UDK 821.131.1’04-39.09Boccaccio G.

\title{
IL SOGNO RACCONTATO NEL FILOCOLO DI GIOVANNI BOCCACCIO
}

\author{
Carmen F. Blanco Valdés
}

\begin{abstract}
The goal of this contribution is to isolate the narrative microtexts referred to dream or sight in such an emblematic work as Il Filocolo, the first great novel by Giovanni Boccaccio, by going into a deep analysis of some aspects like the spatial and temporal situation of the narrative time of the microtext and their subsequent interpretation in the macrotext. On the other hand, it will also be intended to show that the recurrent usage of this kind of microstories is one of the narrative resources used by Boccaccio for upgrading in literary terms the advance of such a long and complex novel; and, thus, it would be convenient to pay attention to the great game of internal references established between the oniric microtexts and the storytelling as a whole.
\end{abstract} testuale

Key words: Boccaccio, narrativa, Filocolo, materia onirica, sagno e visione, microtesto, analisi

In un articolo presentato al Convengo Internazionali di Studi a Banjaluka (Bosnia) su Il sogno italiano (18-19 giugno 2010) in cui centravo lo studio sull'analisi del miscrotesto del sogno in Giovanni Boccaccio (con particolare riguardo a Il Filocolo) da un punto di vista strutturale, si arrivò ad alcune considerazioni che vorrei adesso mettere a fuoco ${ }^{1}$.

In primo luogo, il racconto di cio che possiamo denominare come "materia onirica" s'istaura nella letteratura medioevale come parte integrante di quella realtà cosicché sará assai frequente trovare dei microtesti dei sogni all'interno del macrotesto delle opere. In questo senso, a nostro giudizio, Giovanni Boccaccio diventa, all'interno della tradizione letteraria romanza, il modello letterartio del sogno come microtesto narrativo conferendogli grandi potenzialità produttive.

In secondo luogo, nel Filocolo, la maggior parte dei sogni e, soprattutto, le visioni sono profetiche nel senso che, oltre a servire per informare il lettore di ciò che avverrà posteriormente, motivano letterariamente il racconto, dato che questi microtesti si presentano come l'elemento da cui prende il via lo svolgersi delle azioni posteriori. In

\footnotetext{
${ }^{1}$ Cfr. Blanco Valdés, Carmen F. "Il microtesto del sogno in Giovanni Boccaccio". Convengo Internazionali di Studi "Il sogno italiano", (Banjaluka, 18-20 giugno 2010). Rivista dell'Associazione degli italinisti nei Balcani. (2011). Numero monografíco. In corso di stampa.
} 
questo senso è straordinario, come adesso tenterò di dimostrare, il gioco letterario di riferimenti interni che s'istaura tra i sogni o le visioni e i futuri accadimenti, dimostrando, come poi si è evidenziato nelle sue opere posteriori, il ruolo di primaria importanza che hanno sia i sogni che le visioni nella vita della realtà letteraria creata dal poeta.

Infine, con una struttura narrativa organizzata lungo cinque libri e nella quale $i$ due protagonisti principali, Florio e Biancifiore, si vedono costretti ad essere separati durante il secondo, terzo e quarto libro, l'immaginario narrativo boccacciano doveva trovare un modo di avviare la narrazione mantenendo i contatto la vita e le vicende dei protagonisti: e questo modo è costituito senza dubbio dall'espediente narrativo delle varie visioni e dei sogni che si raccontano, la maggior parte dei quali prende avvio o per invocazione diretta dei personaggi o per intervento diretto degli iddii che si fanno presenti durante il sonno.

L'intenzione di questo studio, come continuazione a quanto detto nel primo articolo, è innanzitutto quella di individuare i sogni e le visioni in una delle opere emblematiche del poeta certaldese qual è Il Filocolo, primo romanzo della letteratura italiana, approfondendo fondamentalmente aspetti come, da un lato, la situazione e il momento narrativo del microtesto del racconto del sogno o visione e, dall'altro, l'interpretabilità, se possibile, del sogno o visione all'interno del macrotesto narrativo.

La struttura narrativa di questo romanzo si svolge lungo cinque libri in cui il primo funziona come prologo all'azione e l'ultimo come epilogo. Lelio e Giulia, genitori di Biancifiore, vanno in pellegrinaggio verso il santuario dell'Apostolo Santiago per ringraziare del figlio che aspettano. Sono attaccati dal saraceno re Felice perché considerati nemici del suo regno. Lelio muore e Giulia viene fatta prigionera e condotta come serva alla corte del re. Florio e Biancifiore nascono lo stesso giorno, giorno della Pascua Fiorita (giustificazione dei nomi). S'innamorano mentre leggono il santo libro d'Ovidio. Quando i genitori di Florio sono informati dell'innamoramento, Florio e Biancifiore sono obbligati a separarsi, vicende che si raccontano lungo il secondo, terzo e quarto libro. I vari episodi in cui viene diviso questo lungo frammento narrativo sono i seguenti: 1.- Biancifiore viene falsamente accusata di tentato omicidio contro il re (Florio riesce a liberarla); 2.- I genitori fanno credere a Florio che Biancifiore ha trovato un nuevo amore nella persona di Fileno (Florio riesce a sapere la vertià dei fatti); 3.- Biancifiore viene venduta ai mercanti che la portano verso Alessandria (i genitori fanno credere a Florio che Biancifiore è morta, ma lui riesce a sapere la vertià, va a cercarla e la trova finalmente); 4.- Florio e Biancifiore si ritrovano finalmente e si sposano, però sono condotti alla morte per tradimento (Ascalion riesce a sapere le circostanze un cui si trovano e li libera). Nel quinto libro, che funziona come abbiamo detto di epilogo, si chiudono alcune delle storie che erano state interrote, tra cui quella di Fileno. Durante il viaggio di ritorno Biancifiore va a Roma dove scopre finalmente la verità del suo lignaggio e vuole ringraziare anche l'Apostolo Santiago portando a fine lo stesso viaggio che i suoi genitori non potessero fare, mentre Florio scopre la vera fede e si fa cristiano essendo coronato a Cordoba come re di un regno ormai cristiano.

La prima domanda che ci dobbiamo porre prima di inziare lo studio concreto dei particolari microtesti narrativi dei sogni in quest'opera è per quali ragioni Boccaccio usa con tanta insistenza questo motivo letterario (un totale di 13 nicrotesti), per poter successivamente arrivare a comprendere la loro funzionalità all'interno del macrotesto. 
E la risposta più inmediata è che, come si vedrá di seguito, sono proprio i sogni o le visioni gli elementi letterari che servono per concatenare i vari episodi narrativi anteriormente descritti e soprattutto quelli che, nella maggior parte dei casi, offrono la soluzione alle varie azioni narrative; e cioè gli incaricati di snodare e chiarire in un determinanto momento l'azione perché la narrazione possa andare avanti.

Nel primo libro di questo romanzo, come abbiamo detto, si racconta la storia dei genitori di Biancifiore, Lelio e Giulia, romani, che vanno in pellegrinaggio al Santuario dell'Apostolo Santiago, nelle lontane coste di Esperia, per ringraziarlo del figlio da tanto tempo desiderato e che ora aspettano. Su istigazione del miserabile re, il cui regno Acheronte circonda, il re Felice, padre di Florio, viene falsamente informato che dei nemici cristiani si stanno dirigendo verso il suo regno per attaccarlo. Sul modello della Farsalia di Lucano si racconta la battaglia in cui Lelio muore e Giulia, incinta di Biancifiore, viene fatta prigionera. Questo è l'inizio che Boccaccio ha ideato per il suo romanzo. Però il motivo che mette in moto questa azione, e di conseguenza la narrazione nel suo complesso, è precisamente la veduta visione di Lelio: il Santo Apostolo che Lelio aveva invocato perché gli concedesse la grazia di avere un figlio facendogli in cambio il voto di visitare i suoi santi luoghi, si fa manifesto e: per me ti manda a dire che il tuo priego è essaudito da Lui, e che, la prima volta che tu con la tua sposa onestamente ti congiungerai, veramente riceverai il dimandato dono. E queste parole dette, ad un'ora egli e'l sonno di Lelio si partirono (Libro I, cap. V). Quando Giulia rimane incinta, allora Lelio capisce che quella visione si è compiuta nel reale e prende la decisione di mettersi in cammino da Roma fino a Santiago, ed è questo il motivo che, come abbiamo visto, dà il via a tutta l'azione posteriore.

L'ultima visione, posta in chiusura del V libro, in questo caso di Biancifiore, si riallaccia direttamente all'episodio della dura battaglia in cui muoiono Lelio e la sua compagnia e in cui Giulia viene fatta prigionera, a ulteriore dimostrazione di quel gioco di riferimenti interni a cui abbiamo prima accennato: Entrati nei padiglioni costoro, $e$ dopo alquanto datisi al sonno, a Biancifiore in fulvida luce un giovane di grazioso aspetto con una giovane bellissima accompagnato, di vermiglio vestiti, le apparvero (Libro $\mathrm{V}$. cap. 90). Con anteriorità a questa visione, Biancifiore e Florio, dopo che quest'ultimo viene incoronato a Cordoba come re di un regno ormai cristiano, vanno anch'essi in pellegrinaggio al santuario dell'Apostolo Santiago, quando per caso si trovano in una profonda valle, la quale tutta d'ossa bianchissime biancheggiava" (Libro, V, cap. 88). Florio e Biancifiore vengono informati che le ossa sono quelle rimaste dopo l'aspra battaglia in cui morì il padre di Biancifiore, ossa tutte mescolate tra le bestie, i cristiani e i saraceni. In questo contesto, i genitori di Biancifiore le si manifestano in visione, e come Lorenzo a Lisabetta nel Decameron, le mostrano esattamente non solo il luogo ma le ossa concrete a cui dovranno dare sacra sepoltura.

È all'interno del lungo macrotesto narrativo del secondo, terzo e quarto libro che si trova la maggior parte dei sogni o visioni, fatto che non ci deve stupire precisamente perchè il punto di vista del narratore, e di conseguenza la storia che si racconta, passa continuamente dall'ottica di Florio a quella di Biancifiore nel corso di questi tre lunghissimi libri.

Il secondo libro si apre con la narrazione dell'innamoramento dei due giovani per intervento diretto di Citerea che invia suo figlio a far innamorare i ragazzi mentre lei 
addormenta il re Felice con un soave sonno durante il quale gli provoca una mirabile visione (Libro II, cap. 5). In effetti, dal momento in cui il loro innamoramento verrà manifestato alla corte del re, la vita dei due diventerà un inferno. In forma allegorica, la visione narra la durezza della vita che aspetta gli amanti, anche se, evidentemente, il re Felice -quantomeno i lettori- non è in grado ancora di dare una corretta interpretazione della visione: vede una cerbia bianca (Biancifiore) aiutata da un leoncello (Florio) perché viene aggredita da un lupo (il senescalo, [Libro II, cap. 28-36]: primo tentativo dei re contro la vita di Biancifiore e difesa fatta da Florio, [Libro II, cap. 59-70]); poi vede, venuti dal mare, due girfalchi (i mercanti a cui è stata venduta Biancifiore e che la portano verso Alessandria, [Libro III, cap. 41-45]) ed infine, dopo che questa cerva è stata inviata in Oriente, un veltro che la vuole anche aggredire (il marsicalo che vuole provacare la morte di Florio e Biancifiore per traizione, [Libro IV, cap. 79]).

In questo momento narrativo inizia la vera espressione artistica dello scrittore che lascerà spazio alla sua creazione letteraria. I giovani sono separati e mentre Florio è lontano dalla corte (sarà inviato dai genitori a Montorio prendendo a giustificazione la sua formazione intellettuale), Biancifiore viene accusata falsamente di tentato omicidio contro il re, con prove ideate dalla propria regina; in questo momento il lettore inizia a interpretare la visione avuta dal re Felice.

Nel secondo libro, in effetti, si narra l'accusa contro Biancifiore e la sua liberazione fatta da Florio stesso, che si presenta a lei travestito. I giovani, come detto, sono già stati separati. La narrazione passa alternativamente da Florio a Biancifiore e da Biancifiore a Florio, il quale si trova in una angoscia permanente per non poter vedere né sapere nulla di Biancifiore. Il tono profetico va in un continuo cescendo narrativo, così come la presenza insistente di un ambiente onirico, unica possibilità di Florio di poter veder attraverso l'immaginazione la sua innamorata, anche se è cosciente della fugacità di questi sogni: posto che tu alcuna volta dormendo io, essendomi tu con benigno aspetto apparita, $m$ 'hai alquanto consolato, la qual consolazione in gravoso tormento s'è voltata, sì tosto com'io mi sveglio dal ingannevole sonno, pensando che veder non ti possa con gli occhi della fronte (Libro II, cap. 41). In questa situazione di soliloquio, Florio invoca gli dei affinché gli concedano l'occasione di poterla vedere prima della propria morte e questi esaudiscono questo desiderio, giusto nel momento in cui: soave sonno gli gravò la testa e, chiusi gli occhi, s'addormentò; e dormendo nuova e mirabile visione gli apparve (Libro II, cap. 41). La dea Venere gli fa vedere con una esattezza cinematografica tutto ciò che è accaduto a Biancifiore nei capitoli precedente [Libro II, cap. 28-36], gli dà dei consigli per la difesa dell'amata e, come prova della sua presenza, gli lascia la spada con cui dovrà difenderla. Quando, rompendosi il debole sonno, si destò e vide nella destra mano la celestiale spada, tutto stupefatto, conobbe essere vero ciò che veduto avea nella preterita visione.

All'inizio del terzo libro Florio, liberata Biancifiore e seguendo il consiglio degli dei, ritornerà a Montorio, mentre lei verrà venduta a dei mercanti e inviata ad Alessandria, dove Florio andrà a cercarla, iniziando così la enquête, la peregrinatio amoris, che occupa la quasi totalità del quarto libro. In questo viaggio il nostro autore si trova, a nostro giudizio, molto a suo agio perché la narrazione del percorso gli offre lo spazio per sviluppare letterariamente tutta la sua immaginazione creativa e fantastica. Il viaggio è dunque una ricreazione letteraria che utilizza il movimento dell'eroe come motivo per 
la narrazione dei fatti del protagonista, facilitando comtemporaneamente l'inserzione di storie parallele all'azione principale.

All'inizio del terzo libro, dunque, quando Florio si separa nuovamente da Biancifiore, Boccaccio farà uso di una nuova allegoria: Florio vede un bianco fiore tutto in mezzo alle spine dei pruni e capisce subito che si tratta della sua Biancifiore. A questo punto la storia improvvisamente ritorna a Biancifiore ed entra in scena l'enigmatica figura di Fileno che dà il via all'elemento narrativo della gelosia che si aggiunge a quello della storia amorosa. Fileno, non a conoscenza dell'amore tra Florio e Biancifiore, si ritrova per caso con il giovane principe e gli dichiara il grande amore che è nato tra lui e una bellisima giovane di nome Biancifiore; e come prova di questo grande amore gli mostra il velo dato da Biancifiore su ordine della regina e contro la volontà della giovane. Cominciano per Florio nuovamente le preocupazioni, tra le quali quella della gelosia. Prende così la decisione di porre fine alla sua miserabile vita. Era ridotto in tale stato che né potea nel suo petto entrar sonno per la continua battaglia de'pensieri e degli abbondanti sospiri (Libro III, cap. 2). In questa situazione di pianto doloroso invoca il valoroso figliuolo di Citerea. La dea Venere scende dal cielo e gli provoca un soavissimo sonno nel quale una mirabile visione gli fu manifesta (Libro III, cap. 19). In questa visione Florio vede l'Amore secondo un'iconografia classica con l'arco e due frecce: una d'oro e un'altra di piombo ${ }^{2}$, con alla sua destra una bellissima donna, che Florio identifica subito con Biancifiore, e alla sua sinistra un mare tempestoso all'interno del quale una nave che procedeva sanza niuno goberno. Nella visione si stabilisce un dialogo a tre tra l'Amore, che gli promette che non lo lascerà perire; Biancifiore, che gli rinfaccia le accuse fatte contro di lei per l'amore nato tra lei e Fileno e gli dà, in segno di pace, un ramo di verde ulivo; e lo stesso Florio. Evidentemente questa visione profetica si riallaccia direttamente all'episodio del naufragio della nave che porta Florio e la sua compagnia alle porte di Partenope [Libro IV, cap. 7]. Allo stesso tempo però serve anche per avviare la narrazione, dato che, da una parte, impedisce che Florio ponga fine alla propria vita, come prima aveva deciso a causa della presunta infedilità di Biancifiore, e dall'altra offre l'opportunità a Biancifiore di chiarire la situazione creatasi con Fileno e la vicenda del velo consegnatogli.

All'interno di questo macrotesto, come abbiamo detto in precedenza, il personaggio di Fileno ricopre un ruolo fondamentale, offrendoci un'altra prova di quel gioco di interferenze interne, tanto care a Boccaccio in quest'opera. Giusto dopo la visione di Florio, la dea Diana, offesa per non essersi trovata nel corteo degli dei che Florio aveva ringraziato dopo la liberazione dell'amata Biancifiore, si dirige all'abitazione della Gelosia per ordinarle che si presenti davanti a Florio e, aprendogli gli occhi gli faccia conoscere com'egli è ingannato (Libro III, cap. 24). Come conseguenza Florio sfodera tutta la sua fierezza contro Fileno al quale desidera di dare la morte. Nuovamente Diana, mossa a compassione di Fileno, si manifesta e si dirige alle case del Sonno riposatore, nascose sotto gli oscuri nuvoli, le quali in lontanissime parti stanno rimote, in una spelonca d'un cavato monte, nella quale Febo con i suoi raggi in niuna maniera può passare (Libro III, cap. 28) per comandargli che si presenti a Fileno e gli faccia conoscere le insidie ordite contro di lui: Mentre che i fatti le cose sinistre così per Fileno

\footnotetext{
${ }^{2}$ Secondo un paso delle Metamorfosi di Ovidio (I, 468-471). Ovidio diceva che il dardo d'oro causava l'amore mentre quello di piombo l'allontanava.
} 
trattavano, Fileno di tutte ignorante si stava pensando alla bellezza di Biancfiore, con sommo disio disiderando quella, quando subito sonno l'assali, e, gli occhi gravati, sopra il suo letto riposandosi si addormentò (...) E così stando, subitamente uno di quelli uficiali in forma d'un caro suo amico gli parve che gli apparisse piangendo e correndo verso lui, e dicessegli: -O Fileno, che fai tu qui? Fuggiti, ch'io ti so dire che l'amore che tu hai portato a Biancifiore t'ha acquistata morte, (Libro III, cap. 30). Comincia allora un altro viaggio, quello dell'esilio di Fileno che alla fine lo porterà in un luogo nel quale, per l'angoscia e il pianto continuo, egli si trasformerà in una fonte e così piangendo e gritando, tutto delle proprie lagrime si bagnava, baciando sovente il candido velo, sopra il quale per debolezza sovente cader si lasciava (Libro III cap. 37). Questo luogo e questa stessa fonte saranno successivamente trovati da Florio durante il suo viaggio alla ricerca di Biancifiore, momento in cui Florio -già Filocolo- viene a conoscenza della storia di Fileno che parla atrraverso la voce che esce dalla fontana: Era nel non conosciuto luogo davanti al vecchio tempio un pratello vestito di pallida erba per la fredda stagione, nel quale una fontana bellisima si vedea (...) Alla quale Filocolo, uscito dal tempio (... ) [Libro IV, cap. 2].

Gli interventi di Diana però non finiscono qui. Furiosa per non aver visto realizzata la vendetta contro gli amanti, si manifesta per la terza volta e in questa occasione lo farà davanti al re Felice, travestita da cacciatrice, per avvisarlo che il figlio desidera togliersi la vita per amore. Prende allora il via un altro episodio fondamentale di questo terzo libro: la decisione del re e della regina di vendere Biancifiore ai mercanti e l'inganno ordito contro Florio a cui fanno credere che Biancifiore sia morta.

Biancifiore si trova sulla nave e inizia il suo viaggio, che però non viene raccontato perché lo si farà attraverso le varie tappe e i vari incontri, pieni di mistero, che Florio avrà durante il suo. In un momento così cruciale della storia non poteva mancare la presenza di uno di questi microtesti: e mentre che Biancifiore queste parole fra sé tacita pregando dicea, soave sonno sopravenutole ... (Libro III, 53). Biancifiore prima di addomentarsi invoca sia la dea Venere, a cui chiede di poter vedere Florio prima di morire, che Diana, di cui si sente un devota fedele, per chiederle di poter servare la propria purezza per il suo innamorato. Entrambe le divinità si fanno presenti durante il sonno per conceder a Biancifiore i doni richiesti e le promettono che tu sarai da Florio ricercata: e in quella parte nella quale più ti parrà impossibile di doverlo potere, avere o vedere, tel troverai nelle tue braccie ignudo. Questo sonno si riallaccia al momento in cui Florio e Biancifiore si incontreranno definitivamente quasi alla fine del quarto libro: Ma Filocolo, che per picciolo pertugio vide nella bella camera entrare Biancifiore, di pietà tale nel viso divenne... [Libro IV, cap. 114]. Però la maestria narrativa dello scrittore non poteva lasciare questo momento così decisivo affidato esclusivamente alla mera narrazione dell'incontro definitivo. Boccaccio vuole dare al macrotesto un risalto particolare e perciò usa -a questo punto non ci dobbiamo stupire- l'espediente narrativo della visione, in questo caso di Glorizia, personaggio che ha accompagnato Biancifiore dall'inizio dell'opera.

Quasi alla fine del quarto libro si racconta, dunque, l'arrivo di Florio ad Alessandria. Una volta che Florio è riuscito a sapere dove si trova la sua Biancifiore, attraverso vari movimenti narrativi il protagonista riesce a introdursi segretamente nella torre dove Biancifiore è custodita. Erroneamente finisce nella stanza di Glorizia che si incarica di 
prepare l'incontro definitivo. Biancifiore è disperata perché vede che le promesse fatte dalle dee in sonno, non si sono realizzate e considera, sconfortata, che gl'iddii come gli uomini abbiano imparato a mentire. A questo punto Glorizia poteva dichiarare semplicemte di aver incontrato Florio, però la maestria narrativa di Boccaccio fa sì che questa glielo comunichi attraverso il racconto di una visione, falsa, avuta la notte precedente. Boccaccio riesce dunque a creare nel lettore l'atmosfera per rendere le visioni più credibili che la stessa realtà dei fatti: Di questo ti rendi certa: che egli vive e amati e cercati, e di qua entro ti trarrà sua, se non mi inganna l'opinione ch'io ho presa d'una nuova visione, che nel sonno di lui e di te questa notte m'apparve" (Libro IV, cap. 113).

Ritornando alla narrazione principale, come abbiamo detto, dopo la separazione degli amanti, inizierà la peregrinatio amoris di Florio, che prenderà da allora il nome di Filocolo, la quale viene raccontata nel corso del quarto libro e all'interno della quale si trova il famoso episodio delle Quistioni d'amore, una delle parti del libro che, senza dubbio, hanno suscitato maggior interesse. Florio-Filocolo si trova a Napoli dove è sopravvissuto a una grande tempesta che ha quasi distrutto la nave che lo portava in cerca di Biancifiore [visione avuta, se rircordiamo, nel Libro III, cap. 18-19]. Lì, mentre aspetta disperato che il tempo migliori, facendo un giorno una passeggiata per la campagna napoletana, ascolta una dolce melodia che lo attira. Lui e la sua compagnia sono invitati a participare a questa gradevole festa nella quale si trova la stessa Fiammetta, che propone, per passare le ore più calde del giorno, che ognuno di loro presenti una questione d'amore che dovrà trovare risposta, gioco questo che si centra sulla logica oppositiva del joc partit di origine provenzale.

Questo quarto libro inizia con un'ambientazione misteriosa e piena di fatti prodigiosi: una soave voce comunica a Florio-Filocolo il percorso che dovrà compiere per trovare la sua innamorata. Tutto il racconto è pieno di sfumature premonitorie in cui le diverse tappe si disegnano attraverso vari indizi che lui trova per caso. In questo ambiente Florio-Filocolo ha una nuova e devastante visione che viene raccontanta a Ascalion, suo caro maestro, dallo stesso Florio retrospettivamente, cioè dopo il suo risveglio: L'accidente della mia turbazione è questo, che nella passata notte io ho veduta la più nuova visione che mai alcuno vedesse, e in quella ho avuto gravissima noia nell'animo, veggendo le cose che io vedea (Libro IV, cap. 12). Attraverso la visione di una povera e bellissima fagiana dietro la quale le stava uno smeriglione e che veniva attaccata da molti altri uccelli provenienti da tutte le parti del mondo, si raccontano in forma allegorica gli avvenimenti che si svolgeranno quasi alla fine del quarto libro, quando gli amanti, una volta che si sono rincontrati, sono scoperti dal mariscalo e sono inviati alla pira per metterli a morte [Libro IV, cap. 126-134]. Inoltre, questo concreto episodio della pira si riallaccia a sua volta a un'altra visione premonitoria, in questo caso di Ascalion, il maestro di Florio, che viene informato del pericolo in cui si trovano i due giovani proprio attraverso l'espediente narrativo della visione durante il sogno: Ma ad Ascalion, quasi più sollecito della salute di Filocolo, entrato di tale stanze in varie imaginazioni, si rivolge per la mente le future cose, e dubitando forte non avvenissero, il tacito sonno con quieto passo gli entra nel petto; e levandolo da quelle, in sé tutto quanto il lega, e nuove e disusate cose gli dimostra, mentre seco il tiene (Libro IV, cap. 136). Non è casuale, a questo punto, che sia precisamente Ascalion colui che ha avuto una visione e che dovrà confermare come vere le cose vedute. Ascalion è uno 
dei personaggi fondamentali in quest'opera perché ricopre la figura dell'uomo saggio, del maestro. È l'interlocutore a cui Florio si rivolge sempre per raccontargli le varie visioni avute. Ascalion reagisce sempre allo stesso modo, non considerando come vere le visioni -come poi capiterà a Gabriotto e alla moglie di Talamo nel Decameron-. Queste sono alcune delle sue opinioni: Non ti recare nella mente sì fatte cose, né dare speranza a'sogni, i quali per poco o per soperchio mangiare, o per imaginazione avuta davanti d'una cosa, sogliono le più volte avvenire, né mai però se ne vide uno vero (Libro II, cap. 44: dopo la prima nuova e mirabile visione di Florio); Manifesta cosa è che ciascuno uomo ne'suoi sonni vede mirabili cose e impossibili e strane, delle quali poi isviluppato si meraviglia, ma conoscendo i principii ove muovono, quelle senza alcun pensiero lascia andare; e però quelle cose che ne conti che vedute hai, sì come vane, nella loro vanità le lascia passare (Libro IV, cap. 14, dopo la piu nuova visione di Florio).

Nel quinto e ultimo libro, dopo le ultime sventure appena raccontate, finalmente Florio si sposa con Biancifore e cominciano il viaggio di ritorno. E se il primo libro funzionava como prologo, quest'ultimo viene ad essere una specie di epilogo, dato che si chiuderanno molte di quelle vicende, strutturate in forma di piccole novelle, che erano stato interrotte durante il viaggio dell'eore, come quella di Fileno, Caleon o Idalogo.

In questo cammino verso la conclusione della storia, Florio avrà un incontro decisivo con frate Ilario, che gli mostrerà il cammino della vera fede. Dopo di che, quando arriveranno nuovamente alla corte del re Felice, questi darà le ultime istruzioni al figlio prima di morire. Alla sua morte Florio verrà incoronato a Cordoba nuovo re di un regno ormai cristiano e compirà il desiderio di visitare il santuario dell' Apostolo Santiago elemento questo che, se ricordiamo, aveva costituito il motivo scatenante di questo romanzo.

L'incontro di Florio con frate Ilario si verifica all'interno di un crescendo narrativo di grande maestria in cui la città di Roma assume il ruolo di protagonista; e lo farà anche attraverso una visione, in questo caso di Biancifiore. Il desiderio della giovane di poter visitare Roma, la sua città natale -anche se lei ancora non lo sa- si fa sempre più forte tanto che chiede che Quello Iddio cui io adoro e in cui io spero, tosto me la faccia vedere. $\mathrm{E}$ in questo momento Biancifiore nel dilicato letto si diede al notturno riposo: la quale poi che de' gradi con che sale ebbe passati cinque, nel sonno furono da Biancifiore mirabili cose vedute (Libro V, cap. 46). In questa visione le appare in forma allegorica la città di Roma sospesa in cielo una donna di grazioso aspetto molto, con da un lato l'Imperatore come simbolo del potere temporale: egli era giovane e robusto e fiero ne'sembianti, e dall'altro lato quello del potere religioso, il Papa: le parea che fosse antico, e negli atti suoi modesto tanto. La donna della visione le dice: però viemmi a vedere sanza alcuno indugio: il tuo fattore vuole, e non sanza gran bene di te e del tuo marito.

Il pellegrino Florio arriva finalmente a Roma e si trova con Ilario che gli domanda come è possibile che lui non conosca la nostra legge. Al che Florio risponde: Male può servare persona la cosa che mai non li fu nota; forse se io questa vostra legge udissi o quello ch 'io dovessi credere mi fosse mostrato, poria essere che, dannando la mia, seguirei questa, e con voi insieme del popolo di Dio diventerei (Libro V, cap. 52). Frate Ilario gli risponde: io la ti mostrerò tutta. È questa la via che porterà alla conversione di 
Florio, anche se il momento veramente iniziale di questo macrotesto narrativo si trova nel libro precedente, quando Florio ha la quinta e ultima delle sue visioni.

In effetti, nel quarto libro, dai capitoli 15 al 72 si svolge, come abbiamo detto, l'episodio delle questioni d'amore. Dopo di ché, nei capitoli inmediatamente successivi vediamo come il macrotesto ci introduce pianamente in una atmosfera di profonda riflessione e introspezione del protagonista. La novità riguardo a questa última visione di Florio è che non si tratta di un visione avuta durante il sogno perché il protagonista è sveglio: Ma ritornato in Partenope, e con malinconia aspettando tempo, avenne che con grandissima malinconia un giorno in un suo giardino si rachiuse solo, e quivi con varii pensieri s'incominciò in se medesimo a dolere, e dolendosi, in nuove cose di pensiero in pensiero il portò la fantasia, portandogli davanti agli occhi, che il loro potere aveano nella mente racconto, nuove e inusitate cose (Libro IV, cap. 74). All'interno di un contesto allegorico e di chiara ispirazione critiana ${ }^{3}$, Florio vede una nave sulla quale si trovano sette donne -come sette sono i doni dello Spirito Santo-, quattro delle quali lui riesce a riconosce: e già d'averle altre fiate vedute e loro contezza avuta si ricordava, -le quattro virtù cardinali-: Prudenza, Giustizia, Fortezza e Temperanza-; mentre che non riesce a sapere chi siano le altre tre, perché, evidentemente, lui ancora non ha mai sentito parlare di loro: Ma le altre tre, che molto più belle gli pareano (...) né quelle per rimirarle in niuno modo conoscere potea. Si tratta delle tre virtù teologali: -Fede, Speranza e Carità- che le sono presentate dalla propria Biancifiore a cui egli indetifica tra le sette donne. In questa particolare visione appare anche un uomo di grandissima eccellenza e autorità che gli racconta molte cose su quelle tre donne che lui non conosce, e finalmente anche una luce mirabilissima, risplendente e grande -rappresentazione della Gloria-come donna bella e graziosa nel aspetto.

Due sono le conseguenze che Florio impara finita questa particolare visione. La prima è che:come questo era fatto, così gli parea aver multiplicata la vista, e meglio conoscere e le mondane cose e le divine che prima, e quelle amare ciacuna secondo il suo dovere. La seconda e più importante per la narrazione del macrotesto è che così sagliendo, gli parea passare infino nelle sante regioni degl'idii. E in quelle conoscere $i$ virtuosi corpi e i loro moti e la loro grandezza e ogni loro potenza: quivi con ammirazione, inestimabile gloria gli parea vedere dalla faccia di Giove procedere a' riguardanti, della quale egli senza fine sentiva. E volendo dire: -Oh felice colui che a tanta gloria è eletto! - avenne che.... Finisce la visione e si produce il trapasso alla realtà con la presenza in scena di Ascalion e Parmenione.

L'incontro con frate Illario, la riscoperta del vero lingaggio di Biancifiore e l'incoronamento di Florio sono degli episodi che si raccontano alla fine di questa storia, in cui il motivo fondamentale è, come abbiamo detto, la conversione di Florio alla vera fede -per la quale è stata decisiva la pseudovisione di Florio-; in modo tale che, quando lo scrittore conclude la narrazione in cui doveva dare dignità alla storia di Florio e Biancifiore, per mandato espresso della musa Fiammetta, il protagonista Florio si ritrova ad aver compiuto il suo itinerario di formazione come perfetto cavalliere -attraverso gli insegnamenti di Ascalion-e come perfetto amante -attraverso le conclusioni avute dalle tredici questioni d'amore-. Coronamento finale della sua formazione di uomo,

\footnotetext{
${ }^{3}$ Fino al punto che in opinioni dei critici tutto l'episodio è elaborato sul modello del Canto XXIX del Purgatorio dantesco.
} 
amante, cavalliere è la conversione alla vera fede che lo farà anche un buon cristiano. Tutto ciò farà di lui il miglior re che al mondo si possa trovare.

Alla luce delle precedenti considerazioni, possiamo giungere ad una conclusione di grande interesse. Nonostante il Filocolo sia considerato, da parte di alcuni critici, un romanzo con una struttura eccessivamente dispersiva, un'opera di gioventù sicuramente erudita in cui, attraverso il procedimento della contaminatio, vengono coinvogliati elementi tratti sia dalla mitologia che da numerosi testi della classicità, ma priva di una originale ispirazione letteraria e di un accurato disegno narrativo prestabilito, tuttavia l'analisi dettagliata dei precisi e strettissimi richiami interni tra i sogni o le visioni e gli avvenimenti che realmente si succedono nella vicenda, ci fanno convinti che, al contrario, Boccaccio aveva sin dall'inizio un disegno chiaro e prestabilito della successione degli avvenimenti secondo una struttura perfettamente pianificata.

Le finalità dell'utilizzo dei microtesti di carattere onirico consistono, a mio parere, da una parte, come accennato in precedenza, nelle possibilità narrative che permettono un dialogo a distanza tra i vari protagonisti del romanzo, che non sarebbe stato possibile realizzare in modo diverso; dall'altra, senza dubbio, il gioco delle anticipazioni delle vicende, attraverso i sogni o le visioni, e il loro successivo disvelarsi nel prosieguo della trama, risponde alla volontà dell'autore di creare e mantenere un clima di mistero e di suspense che alimenti la curiosità del lettore per continuare a leggere un'opera così estesa.

Universitá di Cordoba, Spain

\section{BIBLIOGRAFIA}

Boccaccio, Giovanni. Il Filocolo. Ed. Quaglio, Antonio Enzo. Milano: Mondadori. 1967

Boccaccio, Giovanni, El Filócolo. Trad. e. Ed. Blanco Valdés, Carmen F. Madrid: Gredos. 2004.

Battaglia, Salvatore. "Il primo romanzo della letteratura italiana". La coscienza letteraria del Medioevo. Napoli: Liguori. 1965: 645-657.

Branca, Vittore, "Giovanni Boccaccio rinnovatore dei generi letterari” Atti del Convegno di Nimega sul Boccaccio (28-30 ottobre 1975). Ed. Ballerini, C. Bologna: Pàtron. 1976: 13-25. . Boccaccio medievale. Firenze: Sansoni. 1986.

Bruni, Francesco. "Il Filocolo e lo spazio della letteratura volgare". Boccaccio e dintorni. Miscellanea di studi in onore di Vittore Branca. Firenze: Olschki, 1983: 1-21.

Cazalé-Berard, Claude,"Les structures narratives dans le premier livre du Filocolo de Giovanni Boccaccio". Revue des études italiennes. XVII, 2-3 (1971): 111-132.

Hernández Esteban, María. "Esquemas narrativos del Filócolo". Filología Moderna. XV (1975): 563-581.

Morosini, R. "La 'morte verbale' nel Filocolo. Il viaggio di Florio dall'immaginare al vero consocimento". Studi sul Boccaccio. XXVI (1998): 183-203.

Porcelli, Bruno. "Strutture e forme narrative del Filocolo". Studi sul Boccaccio. XXI (1993): 207233.

Quaglio, Antonio Enzo. "La tradizione del testo del Filocolo". Studi sul Boccaccio. III (1965): 55102.

Testaferri, Ada. "Modello narrativo e semiotica nel Filocolo di Giovanni Boccaccio". Quaderni di italianistica. 8 (1987): 139-148. 